\title{
Response to Induced Relaxation During Pregnancy: Comparison of Women with High Versus Low Levels of Anxiety
}

\author{
Judith Alder • Corinne Urech • Nadine Fink • \\ Johannes Bitzer • Irene Hoesli
}

Published online: 12 January 2011

(C) Springer Science+Business Media, LLC 2011

\begin{abstract}
Relaxation exercises have become a standard intervention for individuals with anxiety disorders but little is known about their potential for anxiety relief during pregnancy. The purpose of this study was to examine psychoendocrine (i) baseline differences and (ii) changes after a standardized relaxation period in pregnant women with high versus low levels of anxiety. Thirty-nine thirdtrimester high and low anxious pregnant women performed active or passive relaxation while levels of anxiety, hypothalamic-pituitary-adrenal (HPA) axis and sympatheticadrenal-medullary (SAM) system activity were assessed before and after the relaxation period. In women with high levels of trait anxiety, state anxiety $(F(1,36)=8.3$, $p=.007)$ and negative affect $(F(1,36)=7.99, p=.008)$ as well as ACTH $(F(1,35)=9.24, p=.002)$ remained elevated over the entire course of the experimental procedure, the last indicating increased HPA axis activity. In addition, norepinephrine showed a constricted decrease of relaxation reflecting lower response of the SAM-system $(F(1,37)=4.41, p=.043)$. Although relaxation exercises have become a standard intervention for individuals with anxiety, pregnant women with high levels of trait anxiety
\end{abstract}

J. Alder $(\square) \cdot$ C. Urech · J. Bitzer · I. Hoesli

Ob/Gyn, University Hospital Basel, Basel, Switzerland

e-mail: jalder@uhbs.ch

\section{N. Fink}

Department of Child and Adolescent Psychiatry and

Psychotherapy, University of Basel, Basel, Switzerland

N. Fink

Children's Hospital, Harvard Medical School, Boston, MA, USA

J. Alder

University Women's Hospital,

Spitalstrasse 21, 4031 Basel, Switzerland benefited less than women with low levels from a single standardized relaxation period.

Keywords Anxiety - Pregnancy - Relaxation exercise · HPA-axis · SAM-system

\section{Introduction}

Research studying the effects of anxiety during pregnancy, its impact on the course of pregnancy and peri/postnatal mental health as well as its implications for treatment has drawn increasing attention in the past decade. Prevalence rates for the presence of any current anxiety disorder during pregnancy lie between 6.6 and 21.7\% (Andersson et al., 2003; Borri et al., 2008; Heron, O'Connor, Evans, Golding, \& Glover, 2004). Most often, specific phobias, followed by social phobias, panic disorders and generalized anxiety disorders (GAD) are diagnosed (Borri et al., 2008; Ross \& McLean, 2006). Methodological differences such as instruments used for the detection of disorders, the studied samples or the use of low cut-off scores to define clinically relevant anxiety account for rather high prevalence ranges. Nevertheless, anxiety disorders are just as prevalent during pregnancy as in non-pregnant women in their reproductive years (Jacobi et al., 2004). While the clinical characteristics are similar to those in pregnant and non-pregnant women, pregnancy and the possible impact on obstetric and fetal outcome constitutes a particular concern (Weisberg \& Paquette, 2002).

In a non-pregnant state, anxiety disorders have been attributed to different neuroendocrine alterations-some of them showing disorder specific patterns. More specifically, dysregulation of noradrenergic neurotransmission has been attributed to the development of anxiety disorders due to its 
effect on cortical and subcortical regions relevant for the processing of fearful stimuli (Neumeister, Daher, \& Charney, 2005). This is reflected by increased activity of the sympathetic-adrenal-medullary (SAM) system and elevated levels of norepinephrine at baseline or reactive to stress challenge in patients with posttraumatic stress disorder (PTSD) (Strawn \& Geracioti, 2008), social phobia (Aouizerate, Martin-Guehl, \& Tignol, 2004), panic disorder (Esler et al., 2006) and GAD (Gorman, Hirschfeld, \& Ninan, 2002). The second major system involved in anxiety is the hypothalamic-pituitary-adrenal (HPA) axis, and there is evidence that abnormalities in HPA axis functioning characterize a subset of anxiety disorders (Mathew, Price, \& Charney, 2008). Comparably, during pregnancy altered neuroendocrine parameters in women with elevated levels of anxiety have been reported by several authors; some findings, however, revealed only modest associations (Field \& Diego, 2008; Field et al., 2003; Obel et al., 2005; Sarkar, Bergman, Fisk, \& Glover, 2006). While physiological stress reactivity appears to be dampened during pregnancy, this might be less the case in women with high levels of stress and anxiety. Thus, in these women, over maternal-placental pathways an excess in cortisol and/or inadequate conversion of active cortisol to inactive cortisone may adversely impact fetal development (de Weerth $\&$ Buitelaar, 2005). The known negative consequence of anxiety during pregnancy on obstetric outcome, fetal and child development among other factors has been attributed to these alterations (Alder, Fink, Bitzer, Hosli, \& Holzgreve, 2007; Federenko \& Wadhwa, 2004; Schneid-Kofman, Sheiner, \& Levy, 2008; Talge, Neal, \& Glover, 2007; Van den Bergh, Mulder, Mennes, \& Glover, 2005).

The management of anxiety disorders during pregnancy is challenged by a lack of sound scientific data clarifying efficacy as well as risk of pharmacotherapy and psychotherapy (e.g., confrontation therapy) (Misri \& Kendrick, 2007; Vythilingum, 2008). Many women are reluctant to take medications during pregnancy and prefer a psychotherapeutic approach. Additionally, the most potent confrontative interventions for the treatment of anxiety syndromes have not been evaluated for their indication during pregnancy. An alternative approach, which is expected to have no harmful side effects is relaxation training with medium to large effect sizes for the reduction of anxiety in non-pregnant individuals (Manzoni, Pagnini, Castelnuovo, \& Molinari, 2008).

There are several studies that have investigated the efficacy of relaxation exercises during pregnancy for different physical and psychological health problems. An early study found relaxation beneficial to pregnant women with elevated levels of blood pressure and a reduction of systolic blood pressure (Little et al., 1984), a finding which has been replicated in normotone patients suffering from bronchial asthma (Nickel et al., 2006). Most but not all studies report on a positive impact of relaxation exercises on intrauterine growth retardation (IUGR), birth weight and being small for gestational age, gestational length and preterm delivery (Bastani, Hidarnia, Montgomery, AguilarVafaei, \& Kazemnejad, 2006; Janke, 1999; Narendran, Nagarathna, Narendran, Gunasheela, \& Nagendra, 2005; Omer, Friedlander, \& Palti, 1986). Similarly, headache improved after relaxation therapy in pregnant patients with migraine or tension-type-headache (Marcus, Scharff, \& Turk, 1995). Psychologically, relaxation exercises and mindfulness-based interventions have lowered levels of anxiety and subjective reports of stress (Bastani, Hidarnia, Kazemnejad, Vafaei, \& Kashanian, 2005; Beddoe \& Lee, 2008; Field et al., 1999; Teixeira, Martin, Prendiville, \& Glover, 2005; Vieten \& Astin, 2008). Few studies have evaluated the effect of relaxation exercises on physiological parameters. Most consistently, a reduction in heart rate has been reported (DiPietro, Costigan, Nelson, Gurewitsch, \& Laudenslager, 2008; Teixeira et al., 2005). On the other hand, an increase in dopamine and a decrease in cortisol and norepinephrine (DiPietro et al., 2008; Field, Diego, Hernandez-Reif, Schanberg, \& Kuhn, 2004; Field et al., 1999; Teixeira et al., 2005) have been observed. Considering the adverse impact of an excessive maternal HPAactivity on fetal development, it could be expected that the physiological benefit of relaxation exercises contributes to the improved pregnancy outcome as reviewed above.

The reported studies differ greatly with regard to the relaxation technique applied, dependent variables and study populations. Most important, none of them addressed the question of whether pregnant women with enhanced levels of anxiety psychologically and physiologically react differently upon relaxation exercises. If however, relaxation techniques as an alternative to psychotropic medication are to be applied to women presenting with symptoms of anxiety during pregnancy, more accurate insight into their relaxation reactivity is essential.

In this paper, a second-order analysis from a randomized controlled trial on the effectiveness of relaxation exercises during pregnancy is presented with the following aims: comparison of the psychoendocrine profile of pregnant women with high and low levels of anxiety (i) at baseline and (ii) in response to a standardized relaxation exercise.

\section{Materials and Methods}

\section{Sample}

The sample consisted of 39 healthy pregnant women which were recruited at the obstetric outpatient department of a university hospital. While waiting for a regular consultation, 
women were approached by a study collaborator, informed about the study and given written informed consent if interested in participation.

To be included, women had to be at least 18 years old, German speaking and pregnant with a single, healthy fetus between the 32 nd -34 th week of gestation. All women had been informed of the healthy state of their fetuses with the last ultrasound realized one to 2 weeks before study participation. Exclusion criteria were intake of glucocorticoids or other medication, severe fetal malformation and preexisting cardiovascular, nephrological, neurological or metabolic diseases. For data analysis, women were allocated to a high anxiety and low anxiety group according to their State-Trait Anxiety Inventory (STAI) trait scores. For a STAI-T cut-off score of 40, Grant et al., (Grant, McMahon, \& Austin, 2008) report a sensitivity of $80.95 \%$ and a specificity of $79.75 \%$ for the presence of an anxiety disorder. The authors propose to apply this score in order to differentiate between women with high and low levels of anxiety. Using this criterion, $28.2 \%$ (11) of the participants were allocated to the high anxiety and $71.8 \%$ (28) to the low anxiety group.

Sample characteristics are shown in Table 1. Groups did not differ with regard to these variables. None of the participants reported being on current treatment with psychotropic medication. High anxious women differed from low anxious women with regard to the number of pregnancy consultations ( 14 vs. 10 consultations, $p<.05$ ). High and low anxious women did not differ with regard to most of the pregnancy outcome parameters (gestational age, birth weight, preterm birth, labor induction, analgesia and complications during delivery), with the exception of fetal distress during delivery, which tended to be more frequent in high anxious women $\left(\chi^{2}=3.53, p=.06\right)$,

Table 1 Sample characteristics $(N=39)$

\begin{tabular}{|c|c|c|}
\hline & Mean $(S D)$ & $\%(n)$ \\
\hline Age & $33.4(4.8)$ & \\
\hline Parity & $0.5(0.7)$ & \\
\hline \multicolumn{3}{|l|}{ Highest degree of education } \\
\hline Compulsory education & & $12.8 \%(5)$ \\
\hline High school & & $7.7 \%(3)$ \\
\hline Vocational school & & $20.5 \%(8)$ \\
\hline Bachelor and master degree & & $59.0 \%(23)$ \\
\hline Married or cohabiting & & $97.4 \%(38)$ \\
\hline \multicolumn{3}{|l|}{ Nationality } \\
\hline Swiss or German & & $76.9 \%(30)$ \\
\hline Other & & $23.1 \%(9)$ \\
\hline Prepregnancy BMI & & $22.3(4.4)$ \\
\hline Prior experience with relaxation exercises & & $28.2 \%(11)$ \\
\hline
\end{tabular}

Study Design and Data Assessment

The study was designed as a randomized controlled trial comparing progressive muscle relaxation (PMR), guided imagery (GI) and passive relaxation (quiet resting) (PR) with regard to psychophysiological reactivity. This secondary outcome analysis evaluated differences in high anxious compared to low anxious women with regard to psychoendocrine baseline characteristics and their response to relaxation.

Women were invited to a $2 \mathrm{~h}$ visit at the hospital in the afternoon and were instructed not to smoke, eat, drink coffee or black tea, nor to do physical exercise $1 \mathrm{~h}$ prior to the appointment. After arrival at the hospital they were asked to go to the bathroom in order to ensure an undisturbed resting period of $2 \mathrm{~h}$. Each woman was then comfortably positioned on the back in a semi-recumbent position followed by a 30-min resting period. Demographic information was assessed and questionnaires on anxiety and positive and negative affect were filled out. In addition, the study midwife applied a permanent catheter for subsequent blood withdrawals. One minute prior to relaxation, subjective levels of relaxation as well as cardiovascular and endocrine measures (pre) were assessed. This pre-testing period was followed by a standardized 10-min period of one of the above mentioned relaxation exercises. The previously recorded tracks were given over headphones and the light in the room was slightly dimmed. One (post1), ten (post10) and twenty (post20) minutes after, subjective levels of relaxation, cardiovascular and endocrine measures were assessed again. In addition, patients re-evaluated their levels of anxiety and positive and negative affect at $20 \mathrm{~min}$ after the procedure.

The research protocol was approved by the regional ethics commission.

\section{Measures}

\section{Psychological Measures}

General demographic, health-related and pregnancy related information (e.g., gestational week, pregnancy complications), as well as information on familiarity with relaxation exercises, was collected during the accommodation period. Two additional items assessed self-reported levels of stress in private and occupational life on a five-point Likert-type scale. In addition, the following two measures were used to investigate anxiety and emotional state:

Spielberger State-Trait Anxiety Inventory. The German version (Laux, Glanzmann, Schaffner, \& Spielberger, 1981) of the STAI (Spielberger, 1983) was applied to measure women's general and situational level of anxiety. Participants were allocated to a high and low anxiety group 
according to their trait-anxiety scores. The STAI shows an internal consistency range from .86 to .95 for the state subscale and from .89 to .91 for the trait subscale. Cronbach's alpha was $>.88$ for state anxiety, and $>.83$ for trait anxiety (Spielberger, 1983). The STAI has been extensively used in experimental studies, including studies with pregnant women (Bastani et al., 2005; Field et al., 2003; Teixeira et al., 2005). For the detection of anxiety disorders during pregnancy the Area under the Curve (AUC) for the trait subscale is .89, indicating that this scale correctly predicted diagnosis/non-diagnosis of antenatal anxiety with a probability of $89.2 \%$ (Grant et al., 2008). These authors therefore propose the use of a cut-off score of $>40$ to identify pregnant women with a high probability of meeting the diagnostic criteria of an anxiety disorder.

Positive and Negative Affect Schedule (PANAS, Watson, Clark, \& Tellegen, 1988). This scale consists of 10 items to assess positive (PA) and 10 items to assess negative affect (NA). Participants are asked to rate the affect items on a scale from 1 to 5 , based on the strength of emotion $(1=$ "very slightly or not at all" and $5=$ "extremely"). The scale has been shown to be highly internally consistent and the two subscales are largely uncorrelated. Internal consistency ranges from .86 to .90 for positive affect and .84 to .87 for negative affect.

Before and after the relaxation exercise (pre-relaxation, post-relaxation 1, post-relaxation 2, post-relaxation 3) participants were asked to rate their experienced level of relaxation on a visual analogue scale (VAS) consisting of a horizontal line, $100 \mathrm{~mm}$ in length, ranging from "not at all relaxed" at the left end to "extremely relaxed" at the right end. Participants marked on the line the point that represent their perception of the current state. The VAS score could range from 0 to 10 and was determined by measuring in centimeters (with millimeter accuracy) the distance from the left hand end of the line to the point that the participant marked.

\section{Physiological Measures}

Endocrine sampling methods and biochemical analyses. Blood samples were collected at four time points (pre, post1, post10, post20) using EDTA-monovettes with anticoagulant for plasma. Immediately after sampling, bloodplasma samples were centrifuged at 3000 rotations $/ \mathrm{min}$ for $10 \mathrm{~min}$ at $4^{\circ} \mathrm{C}$ and stored in test tubes at $-80^{\circ}$ until analysis. For cortisol sampling, participants were asked to chew on a bite-sized piece of paraffin wax to produce drooling (Odusola, 1991). Saliva was then collected into $2 \mathrm{ml}$ tubes and stored at $-20^{\circ} \mathrm{C}$ until analysis.

The hormonal assays were conducted at the biochemical laboratory of the University of Trier, Germany. For the quantitative determination of cortisol in saliva, a time-resolved fluorescence immunoassay (TF-FIA) was performed. TF-FIA showed excellent correlation with a commercially available radioimmunoassay kit for salivary cortisol measurement (Dressendorfer, Kirschbaum, Rohde, Stahl, \& Strasburger, 1992). Variation coefficient for intra-assay variation ranged between $4.0-6.7 \%$, and $7.1-9.0 \%$ for inter-assay variation. Recovery test scores ranged from 91 to $106 \%$.

Adrenocorticotropin (ACTH) was determined using the ACTH Immunoassay Kit (SG51041). It is a two-site ELISA for the measurement of the biologically active 39 amino acid chain of ACTH and shows a cross reactivity of $-5.7 \%$ to low amounts $(200 \mathrm{pg} / \mathrm{ml})$ of added $\alpha$-Melanotropine. No further limitations of the specificity $(>.01)$ are reported. In recovery tests where known amounts of ACTH were added to a previously assayed serum sample, the kit detected on average $97 \%$ of the added ACTH. Controls were run on every plate for determination of inter- and intra-assay variability, which was $<7 \%$ for this procedure.

Norepinephrine (NE) and epinephrine (E) in plasma were assigned with the ClinRep ${ }^{\circledR}$ Complete Kit (Cat.-Nr.: 1000 ), which is based on high performance liquid chromatography (HPLC) according to the method of Kringe, Neidhardt and Lippmann (1982). Intra-assay variation is expected to be around $8 \%$.

Cardiovascular responses. Maternal cardiovascular response was measured four times (pre-relaxation, postrelaxation 1, post-relaxation 2, post-relaxation 3) with a GE DINAMAP ${ }^{\circledR}$ ProCare 400. It records systolic (SBP, $\mathrm{mmHg}$ ) and diastolic (DBP, $\mathrm{mmHg}$ ) blood pressure as well as heart rate (HR, bpm).

\section{Pregnancy Outcome}

Information on pregnancy outcome was extracted from patient files after delivery. The following information was used for the present analyses: gestational age, birth weight, head circumference, umbilical cord $\mathrm{pH}$ values, intake of medication during pregnancy (dichotomous), analgesics and anesthesia during delivery (dichotomous), fetal distress (dichotomous, defined as $\mathrm{pH}$-value below 7.20, pathologic cardiotocogram and meconium in the amniotic fluid) and labor induction (dichotomous). In addition, number of pregnancy consultations was assessed and number of pregnancy complications (diagnosis of preterm contractions, hyperemesis, gestational diabetes, gestational hypertension and proteinuria) was summarized to an obstetric complications sum score (OCSS).

\section{Data Analysis}

As the three experimental conditions did not differ with regard to endocrine reactivity (Urech et al., 2010), the type 
of relaxation was not considered any further in the analysis. After log-transformation, Cortisol, NE and $\mathrm{E}$ values met the criteria for normal distribution. Baseline differences in mean values of $\mathrm{E}, \mathrm{NE}$, cortisol and $\mathrm{ACTH}$, cardiovascular parameters and psychological variables (positive and negative affect, state anxiety and levels of stress in private and occupational life) in patients with high versus low levels of anxiety were investigated by Student's $t$-tests.

To investigate pre-post group differences in psychological and endocrine variables, repeated measures analysis of variance (ANOVA) with a twofold factor Group (high/ low anxiety) was calculated. For $\mathrm{E}$ and $\mathrm{NE}$ and the psychological variables within-subject factor Time was twofold (for hormones $1 \mathrm{~min}$ pre, $1 \mathrm{~min}$ post and for psychological measures pre and $20 \mathrm{~min}$ post); while for ACTH and cortisol the Time factor was fourfold with additional measuring time points at 10 and 20 min post relaxation. Greenhouse Geisser correction of degrees of freedom was applied if assumption of sphericity was violated. To test for between-subject effects the standard setting of the quadratic sum type III was retained to account for unequal group sizes. Effect sizes are reported as partial Eta squared $\left(\eta_{\mathrm{p}}^{2}\right)$.

All statistical analyses were conducted using SPSS 16.0.2 (SPSS Inc., Chicago, USA) and $p$-levels of $<.05$ were considered significant.

Table 2 Psychological and physiological baseline values in women with high compared to low levels of anxiety

\begin{tabular}{lccc}
\hline Variable & $\begin{array}{l}\text { High levels } \\
\text { of anxiety } \\
\text { Mean }(S D)\end{array}$ & $\begin{array}{l}\text { Low levels } \\
\text { of anxiety } \\
\text { Mean }(S D)\end{array}$ & $T$-value \\
\hline Private stress & $3.3(1.4)$ & $1.8(0.8)$ & $4.12^{* * * *}$ \\
Occupational stress & $2.3(1.3)$ & $2.3(1.0)$ & -0.03 \\
STAI-state & $38.9(12.1)$ & $31.2(4.6)$ & $2.9^{* *}$ \\
STAI-trait & $48.5(10.6)$ & $30.2(4.4)$ & $7.7^{* * *}$ \\
Positive affect & $33.3(4.7)$ & $34.5(5.8)$ & -0.74 \\
Negative affect & $16.3(6.9)$ & $12.2(2.9)$ & $2.6 *$ \\
Cortisol & $7.9(3.0)$ & $7.3(5.5)$ & 0.97 \\
ACTH & $40.9(26.5)$ & $27.6(12.8)$ & $2.1 *$ \\
E & $38.5(23.1)$ & $34.6(20.2)$ & 0.24 \\
NE & $242.3(136.4)$ & $245.1(83.6)$ & -0.50 \\
SBP & $98.3(10.3)$ & $103.4(14.9)$ & -1.10 \\
HR & $83.1(9.4)$ & $78.7(14.3)$ & 0.93 \\
\hline
\end{tabular}

$\overline{A C T H}$ adrenocorticotropin, $E$ epinephrine, $N E$ norepinephrine, $S B P$ systolic blood pressure, $H R$ heart rate

$* p<.05, * * p<.01, * * * p<.001$

\section{Results}

Baseline Parameters in Women with High and Low Levels of Anxiety

High anxious were compared to low anxious women with regard to their baseline psychological and physiological parameters. Table 2 displays mean values, standard deviations and $T$-values for group comparison of mean values. Anxious women reported higher levels of private stress $(T=4.12, p<.001)$, higher state anxiety scores $(T=2.9$, $p<.01)$ and more negative affect $(T=2.6, p<.05)$. The differences in levels of ACTH $(T=2.1, p<.05)$ reflect an increased baseline activity of the HPA-axis in highly anxious compared to low anxious women. Interestingly, only trait- but not state-anxiety scores were correlated with enhanced HPA-activity at baseline.

Psychoendocrine Reactivity in Women with High and Low Levels of Anxiety

To compare endocrine reactivity upon relaxation in high anxious versus low anxious women, levels of epinephrine, norepinephrine, cortisol, and ACTH were investigated over the course of the experimental procedure. As the time effect of the endocrine parameters did not vary between the three different exercises, we did not additionally control for relaxation technique. Figure 1 shows the course of the hormones over time for the two groups. Levels of epinephrine did not differ between groups and there was no interaction of Time and Group. However, for norepinephrine a significant interaction effect between Time and Group $\left(F(1,37)=4.41, p=.043, \eta_{\mathrm{p}}^{2}=.107\right)$ and a time effect $\left(F(1,37)=12.47, \quad p=.001, \quad \eta_{\mathrm{p}}^{2}=.252\right) \quad$ was observed (see Fig. 1). This indicates that while both groups did react with a decrease of norepinephrine levels, this was significantly less pronounced for the group with high levels of anxiety. For cortisol, only a time effect $(F(2.08$, $\left.35)=8.17, p=.001, \eta_{\mathrm{p}}^{2}=.181\right)$ was observable while groups did not differ. ACTH-levels did change over time $\left(F(1.28,33)=4.23, p=.036, \eta_{\mathrm{p}}^{2}=.108\right)$. In addition, a significant group effect was found, with women in the high anxiety group showing higher ACTH levels over the entire course of the study $\left(F(1,35)=9.24, p=.002, \eta_{\mathrm{p}}^{2}=.209\right)$.

For PANAS negative affect, a significant time $\left(F(1,36)=8.16, \quad p=.007, \quad \eta_{\mathrm{p}}^{2}=.185\right) \quad$ and betweensubject effect $\left(F(1,36)=7.99, p=.008, \eta_{\mathrm{p}}^{2}=.182\right)$ was observed with women with high levels of anxiety reporting more negative affect. However, the non-significant interaction effect shows that high and low anxious women did not benefit differently from the relaxation period with regard to their negative affect. The same applied to levels of state anxiety in the STAI, where a highly significant 
Fig. 1 Means (SE) of the endocrine variables before (pre; $1 \mathrm{~min}$ ) and after (post 1 , post 10 and post $20 \mathrm{~min}$ ) the relaxation period for women with high and low levels of anxiety
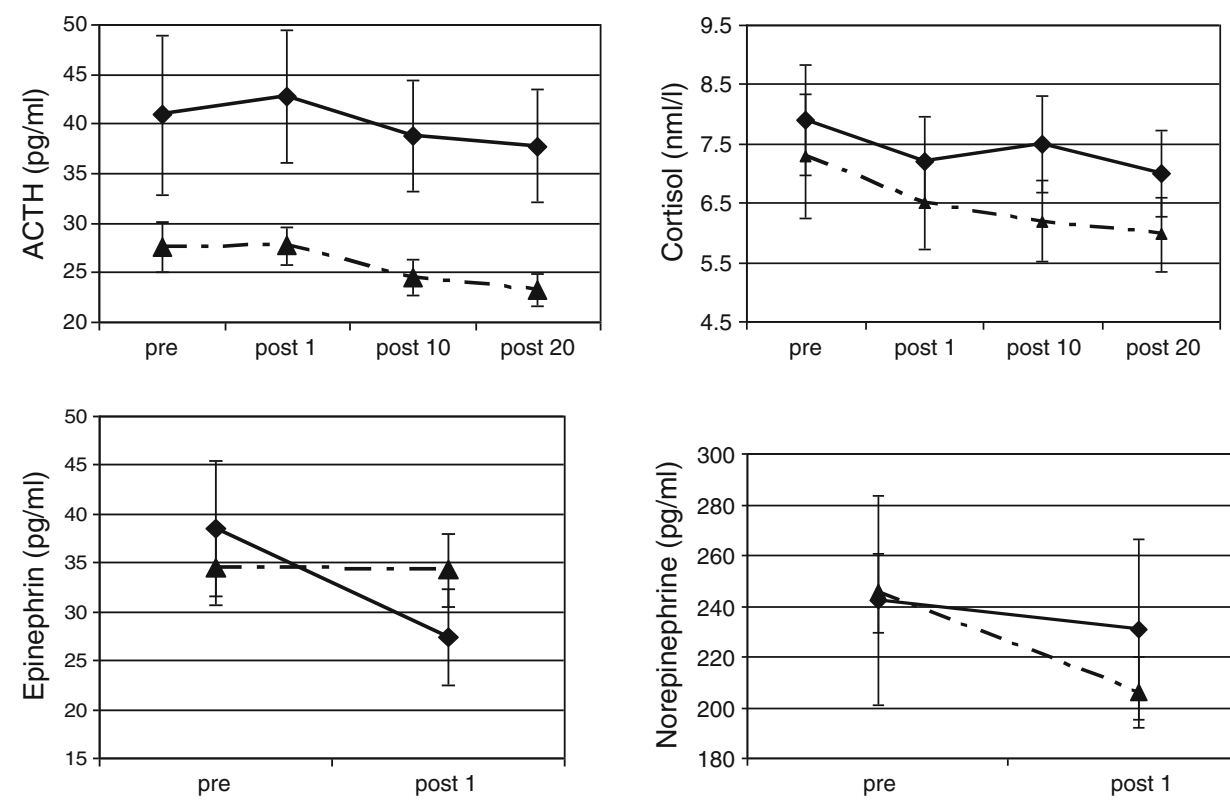

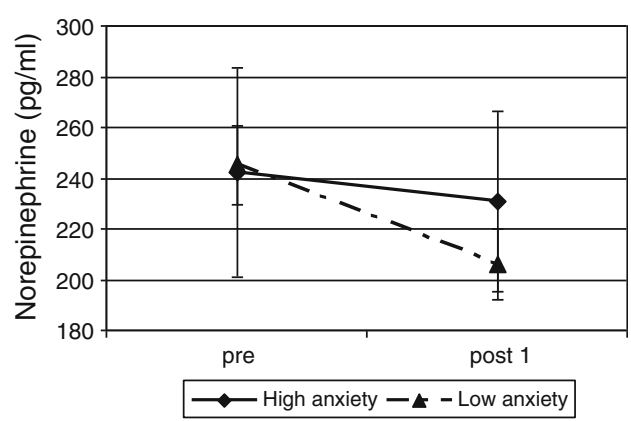

time effect $\left(F(1,36)=12.88, p=.001, \eta_{\mathrm{p}}^{2}=.264\right)$ and a group effect $\left(F(1,36)=8.3, p=.007, \eta_{\mathrm{p}}^{2}=.188\right)$ but no interaction effect were observed.

With regard to self-rated levels of relaxation, VAS-scores in both groups indicated higher levels of relaxation after the relaxation procedure for all time points (post 1: $\left.F(1,37)=54.03, p=.000, \eta_{\mathrm{p}}^{2}=.594\right)$; post $10: F(1,37)=$ $20.06, p=.000, \eta_{\mathrm{p}}^{2}=.352$; post $20: F(1,37)=23.15$, $\left.p=.000, \eta_{\mathrm{p}}^{2}=.385\right)$. A group effect was found for the VAS scores prior to the relaxation lower scores in the high anxiety group. However, no interaction effect was observed.

Psychologically, the relaxation procedure therefore showed an effect from which high anxious women seemed to benefit similarly to low anxious women.

\section{Discussion and Conclusions}

The present study's aims were to assess the psychophysiological baseline characteristics in women with high and low levels of anxiety and to compare the endocrine reactivity upon relaxation in these two groups. The results suggest an increased activity of the HPA axis in pregnant women with high levels of anxiety, with significantly augmented levels of ACTH. Levels of cortisol were nonsignificantly elevated. In addition to limited power due to rather small sample size this may be the consequence of higher context dependency of cortisol (nutrition, sleep pattern, time of day). Baseline samples were extracted after a waiting period of $30 \mathrm{~min}$ to account for these individual differences; this may, however, also have washed out some of the effects of the standardized relaxation procedure. On the other hand, women may differ in their perception of the catheterization as being painful and aversive which might influence the baseline endocrine values. However, VAS scores, which were used to assess level of pain after the catheter was installed, displayed no association of pain experience and endocrine baseline measures or level of trait anxiety. The observed group differences in baseline levels may therefore not be a result of differences in pain experience.

Also, even though all of the measurements took place in the afternoons there was still variability in time of about $3 \mathrm{~h}$. Women were asked not to eat and drink $1 \mathrm{~h}$ before their appointment but we did not standardize meals on the experimental day and relied on self-reports.

Interestingly, in women with high levels of anxiety, the HPA-hormones were not correlated while in women with low levels of anxiety, ACTH and cortisol did significantly correlate with each other. This is a finding which has previously been reported by Talley, Heitkemper, Chicz-Demet and Sandman (2006) for pregnant women in abusive relationships. Comparably, ACTH and cortisol values in battered women did not correlate while they showed a clear linear relationship in non-battered women. The authors concluded that the experience of stress in this population altered the relationship of HPA-axis hormones perhaps resulting in an exhaustion of the adrenal glands incapable of mounting a response to ACTH.

An alteration of HPA-activity has been reported also for patients with anxiety disorders outside of pregnancy with certain disorder specific patterns (Mathew et al., 2008). The present study does not identify type of anxiety disorder nor is the use of a STAI-trait cut-off score valid to make a 
diagnostic confirmation of the presence or absence of an anxiety disorder. Nevertheless, as suggested by Grant et al. (2008) the probability of a correct prediction of an anxiety disorder/no anxiety disorder for the applied cut off score is almost as high as $90 \%$. Field et al. (2001) reported similar findings for pregnant women with co-morbidly enhanced levels of anxiety and depression. While they did not study levels of ACTH, they found higher levels of cortisol and dopamine. Comparable to our results, no baseline differences were found for epinephrine and norepinephrine. In addition, for specific populations such as women awaiting amniocentesis, where enhanced levels of state anxiety have been observed, levels of cortisol were related to anxiety. Again, these authors did not report on levels of ACTH (Sarkar et al., 2006). Some authors found enhanced levels of ACTH and/or cortisol in pregnant women with high levels of stress (Obel et al., 2005; Sandman, Wadhwa, Chicz-DeMet, Dunkel-Schetter, \& Porto, 1997) or for comorbid conditions (Evans, Myers, \& Monk, 2008). The present results therefore support the hypothesis of a change in the HPA-axis anxiety-response threshold during pregnancy. Therefore, while pregnancy in general has been associated with an improved stress "immune" state, it may not apply to distressed and anxious women (de Weerth \& Buitelaar, 2005).

Most important, however, these differences were not limited to baseline measurements but were also present after a period of relaxation. One important scope of this secondary outcome analysis was to determine the course of endocrine patterns from baseline to $20 \mathrm{~min}$ after relaxation in women with high compared to low levels of anxiety. While comparable to others (Teixeira et al., 2005), HPA-axis hormones did decrease over time, the noted group differences in ACTH remained over the course of the procedure. Therefore, women from the high anxiety group did not benefit from the relaxation period by an additional harmonization of the HPA-axis hormones. Also, the differences between high and low anxious women were even more crucial for norepinephrine: The significant interaction effect indicates that levels of norepinephrine decreased in women with high compared to those with low levels of anxiety significantly less.

In addition, we did not observe an anxiety-specific psychological benefit. While in both groups levels of state anxiety and negative affect decreased from before to after the intervention, there was no additional improvement in women with high levels of anxiety.

Several explanations may relate to these observations. The primary scope of the study was to pilot the use of relaxation techniques and evaluate their psychobiological actions. Even though the relaxation period was limited to a 10 min-period, this secondary analysis showed that women with high levels of anxiety had less pronounced reactions during these one-time exercises and they may need more counselling, instructions and practice than low anxious women. Also, it may be that high anxious women need more time to relax and, thus, the $10 \mathrm{~min}$ period may have been too short for these women to develop the same psychological and physiological benefits as their low anxious counterparts.

Future studies will therefore have to focus more differentially on mid- to long-term effects of repeated relaxation exercises during pregnancy on psychoendocrine parameters in women with high levels of anxiety. Several authors have found a psychological (Bastani et al., 2005; Field, Hernandez-Reif, Diego, Schanberg, \& Kuhn, 2005; Field et al., 1999) and physiological (Field et al., 1999) benefit from repeated relaxation; however they did not look for separate effects in women suffering from anxiety. Especially since relaxation training is a standard intervention in anxiety patients, it is of major importance to understand the psychophysiological mechanisms in reducing symptoms of anxiety during pregnancy.

Notably, the utility of relaxation training to positively affect obstetric outcome is an additional field, which has received only little attention in the scientific literature. Anxiety during pregnancy has been associated with adverse obstetric outcome (Alder et al., 2007), but few studies have systematically evaluated the benefit of relaxation training in these patient groups to improve obstetric variables. While some authors have previously reported associations between anxiety or mixed conditions and obstetric complications (Andersson et al., 2003; Andersson, SundstromPoromaa, Wulff, Astrom, \& Bixo, 2004; Kurki, Hiilesmaa, Raitasalo, Mattila, \& Ylikorkala, 2000), others have not found such an association (Field et al., 2003). Further research will, however, not only have to look for the obstetric risks of highly anxious women but also understand better the pathways by which anxiety contributes to obstetric complications. Moreover, the psychophysiological potency of regularly performed relaxation exercises to improve obstetric outcome has yet to be elucidated systematically.

Another limitation of this study which has to be addressed further is a potential sample bias with the majority of women being highly educated, in stable relationships and a comfortable socioeconomic situation and therefore not representative of a general pregnant population. Nevertheless, this is the first study to show that women with high levels of anxiety do not respond as sensitively to relaxation as low anxious women do, and this needs to be considered when counselling women presenting with anxiety symptoms during pregnancy. If relaxation exercises are to be imparted as part of a counselling intervention, high anxious women should be monitored carefully; issues and questions by patients should be 
rediscussed in the next consultation. Ideally, objective measures (physiologic response) should complement evaluation of subjective effectiveness.

Acknowledgments This work is part of the National Centre of Competence in Research (NCCR) Swiss Etiological Study of Adjustment and Mental Health (sesam).The Swiss National Science Foundation (SNF) (project no. 51A240-104890), the University of Basel and the Freie Akademische Gesellschaft provided core support for the NCCR sesam. This publication is the work of the authors. Judith Alder, Ph.D. serves as guarantor for the paper. This research was specifically supported by the University Women's Hospital of Basel. We are grateful to the biochemical laboratory of the University of Trier, Germany for the analyses of blood and saliva samples and for their expert advice.

\section{References}

Alder, J., Fink, N., Bitzer, J., Hosli, I., \& Holzgreve, W. (2007). Depression and anxiety during pregnancy: A risk factor for obstetric, fetal and neonatal outcome? A critical review of the literature. Journal of Maternal-Fetal and Neonatal Medicine, 20, 189-209.

Andersson, L., Sundstrom-Poromaa, I., Bixo, M., Wulff, M., Bondestam, K., \& Astrom, M. (2003). Point prevalence of psychiatric disorders during the second trimester of pregnancy: A population-based study. American Journal of Obstetrics and Gynecology, 189, 148-154.

Andersson, L., Sundstrom-Poromaa, I., Wulff, M., Astrom, M., \& Bixo, M. (2004). Implications of antenatal depression and anxiety for obstetric outcome. Obstetrics and Gynecology, 104, 467-476.

Aouizerate, B., Martin-Guehl, C., \& Tignol, J. (2004). Neurobiology and pharmacotherapy of social phobia. Encephale, 30, 301-313.

Bastani, F., Hidarnia, A., Kazemnejad, A., Vafaei, M., \& Kashanian, M. (2005). A randomized controlled trial of the effects of applied relaxation training on reducing anxiety and perceived stress in pregnant women. Journal of Midwifery and Women's Health, 50, e36-e40.

Bastani, F., Hidarnia, A., Montgomery, K. S., Aguilar-Vafaei, M. E., \& Kazemnejad, A. (2006). Does relaxation education in anxious primigravid Iranian women influence adverse pregnancy outcomes?: A randomized controlled trial. Journal of Perinatal and Neonatal Nursing, 20, 138-146.

Beddoe, A. E., \& Lee, K. A. (2008). Mind-body interventions during pregnancy. Journal of Obstetric, Gynecologic, and Neonatal Nursing, 37, 165-175.

Borri, C., Mauri, M., Oppo, A., Banti, S., Rambelli, C., Ramacciotti, D., et al. (2008). Axis I psychopathology and functional impairment at the third month of pregnancy: Results from the Perinatal Depression-Research and Screening Unit (PND-ReScU) Study. Journal of Clinical Psychiatry, 69(19), 1617-1624.

de Weerth, C., \& Buitelaar, J. K. (2005). Physiological stress reactivity in human pregnancy-A review. Neuroscience and Biobehavioral Reviews, 29, 295-312.

DiPietro, J. A., Costigan, K. A., Nelson, P., Gurewitsch, E. D., \& Laudenslager, M. L. (2008). Fetal responses to induced maternal relaxation during pregnancy. Biological Psychology, 77, 11-19.

Dressendorfer, R. A., Kirschbaum, C., Rohde, W., Stahl, F., \& Strasburger, C. J. (1992). Synthesis of a cortisol-biotin conjugate and evaluation as a tracer in an immunoassay for salivary cortisol measurement. Journal of Steroid Biochemistry and Molecular Biology, 43, 683-692.
Esler, M., Alvarenga, M., Pier, C., Richards, J., El-Osta, A., Barton, D., et al. (2006). The neuronal noradrenaline transporter, anxiety and cardiovascular disease. Journal of Psychopharmacology, 20, 60-66.

Evans, L. M., Myers, M. M., \& Monk, C. (2008). Pregnant women's cortisol is elevated with anxiety and depression-But only when comorbid. Archives of Women's Mental Health, 11, 239-248.

Federenko, I. S., \& Wadhwa, P. D. (2004). Women's mental health during pregnancy influences fetal and infant developmental and health outcomes. CNS Spectrums, 9, 198-206.

Field, T., \& Diego, M. (2008). Cortisol: The culprit prenatal stress variable. International Journal of Neuroscience, 118, 1181.

Field, T., Diego, M. A., Hernandez-Reif, M., Schanberg, S., \& Kuhn, C. (2004). Massage therapy effects on depressed pregnant women. Journal of Psychosomatic Obstetrics and Gynaecology, $25,115-122$.

Field, T., Diego, M., Hernandez-Reif, M., Schanberg, S., Kuhn, C., Yando, R., et al. (2003). Pregnancy anxiety and comorbid depression and anger: Effects on the fetus and neonate. Depression and Anxiety, 17, 140-151.

Field, T., Hernandez-Reif, M., Diego, M., Schanberg, S., \& Kuhn, C. (2005). Cortisol decreases and serotonin and dopamine increase following massage therapy. International Journal of Neuroscience, 115, 1397-1413.

Field, T., Hernandez-Reif, M., Hart, S., Theakston, H., Schanberg, S., \& Kuhn, C. (1999). Pregnant women benefit from massage therapy. Journal of Psychosomatic Obstetrics and Gynaecology, 20, 31-38.

Field, T., Miguel, A. D., Dieter, J., Hernandez-Reif, M., Schanberg, S., Kuhn, C., et al. (2001). Depressed withdrawn and intrusive mother's effects on their fetuses and neonates. Infant Behavior \& Development, 24, 27-39.

Gorman, J. M., Hirschfeld, R. M., \& Ninan, P. T. (2002). New developments in the neurobiological basis of anxiety disorders. Psychopharmacology Bulletin, 36, 49-67.

Grant, K. A., McMahon, C., \& Austin, M. P. (2008). Maternal anxiety during the transition to parenthood: A prospective study. Journal of Affective Disorders, 108, 101-111.

Heron, J., O’Connor, T. G., Evans, J., Golding, J., \& Glover, V. (2004). The course of anxiety and depression through pregnancy and the postpartum in a community sample. Journal of Affective Disorders, 80, 65-73.

Jacobi, F., Wittchen, H. U., Holting, C., Hofler, M., Pfister, H., Muller, N., et al. (2004). Prevalence, co-morbidity and correlates of mental disorders in the general population: Results from the German Health Interview and Examination Survey (GHS). Psychological Medicine, 34, 597-611.

Janke, J. (1999). The effect of relaxation therapy on preterm labor outcomes. Journal of Obstetric, Gynecologic, and Neonatal Nursing, 28, 255-263.

Kringe, K. P., Neidhardt, B., \& Lippmann, C. M. (1982). Practical aspects of the routine determination by HPLC of free noradrenaline and adrenaline in urine and plasma. In I. Mohnar (Ed.), Practical aspects of modern HPLC. New York: De Gryter.

Kurki, T., Hiilesmaa, V., Raitasalo, R., Mattila, H., \& Ylikorkala, O. (2000). Depression and anxiety in early pregnancy and risk for preeclampsia. Obstetrics and Gynecology, 95, 487-490.

Laux, L., Glanzmann, P., Schaffner, P., \& Spielberger, C. D. (1981). STAI; State-trait-Angstinventar; State-Trait Anxiety Inventory. Weinheim: Beltz.

Little, B. C., Hayworth, J., Benson, P., Hall, F., Beard, R. W., Dewhurst, J., et al. (1984). Treatment of hypertension in pregnancy by relaxation and biofeedback. Lancet, 1, 865-867.

Manzoni, G. M., Pagnini, F., Castelnuovo, G., \& Molinari, E. (2008). Relaxation training for anxiety: A ten-years systematic review with meta-analysis. BMC Psychiatry, 8, 41. 
Marcus, D. A., Scharff, L., \& Turk, D. C. (1995). Nonpharmacological management of headaches during pregnancy. Psychosomatic Medicine, 57, 527-535.

Mathew, S. J., Price, R. B., \& Charney, D. S. (2008). Recent advances in the neurobiology of anxiety disorders: Implications for novel therapeutics. American Journal of Medical Genetics. Part C, Seminars in Medical Genetics, 148, 89-98.

Misri, S., \& Kendrick, K. (2007). Treatment of perinatal mood and anxiety disorders: A review. Canadian Journal of Psychiatry, 52, 489-498.

Narendran, S., Nagarathna, R., Narendran, V., Gunasheela, S., \& Nagendra, H. R. (2005). Efficacy of yoga on pregnancy outcome. Journal of Alternative and Complementary, 11, 237-244.

Neumeister, A., Daher, R. J., \& Charney, D. S. (2005). Anxiety disorders: Noradrenergic neurotransmission. Handbook of Experimental Pharmacology (169), 205-223.

Nickel, C., Lahmann, C., Muehlbacher, M., Pedrosa Gil, F., Kaplan, P., Buschmann, W., et al. (2006). Pregnant women with bronchial asthma benefit from progressive muscle relaxation: A randomized, prospective, controlled trial. Psychotherapy and Psychosomatics, 75, 237-243.

Obel, C., Hedegaard, M., Henriksen, T. B., Secher, N. J., Olsen, J., \& Levine, S. (2005). Stress and salivary cortisol during pregnancy. Psychoneuroendocrinology, 30, 647-656.

Odusola, F. (1991). Chewing gum as aid in treatment of hyposalivation. New York State Dental Journal, 57, 28-31.

Omer, H., Friedlander, D., \& Palti, Z. (1986). Hypnotic relaxation in the treatment of premature labor. Psychosomatic Medicine, 48, $351-361$.

Ross, L. E., \& McLean, L. M. (2006). Anxiety disorders during pregnancy and the postpartum period: A systematic review. Journal of Clinical Psychiatry, 67, 1285-1298.

Sandman, C. A., Wadhwa, P. D., Chicz-DeMet, A., Dunkel-Schetter, C., \& Porto, M. (1997). Maternal stress, HPA activity, and fetal/ infant outcome. Annals of the New York Academy of Sciences, $814,266-275$.

Sarkar, P., Bergman, K., Fisk, N. M., \& Glover, V. (2006). Maternal anxiety at amniocentesis and plasma cortisol. Prenatal Diagnosis, 26, 505-509.

Schneid-Kofman, N., Sheiner, E., \& Levy, A. (2008). Psychiatric illness and adverse pregnancy outcome. International Journal of Gynaecology and Obstetrics, 101, 53-56.
Spielberger, C. D. (1983). Manual for the State-Trait Anxiety Inventory (STAI). Palo Alto, CA: Consulting Psychologists Press.

Strawn, J. R., \& Geracioti, T. D., Jr. (2008). Noradrenergic dysfunction and the psychopharmacology of posttraumatic stress disorder. Depression and Anxiety, 25, 260-271.

Talge, N. M., Neal, C., \& Glover, V. (2007). Antenatal maternal stress and long-term effects on child neurodevelopment: How and why? Journal of Child Psychology and Psychiatry, 48, 245-261.

Talley, P., Heitkemper, M., Chicz-Demet, A., \& Sandman, C. A. (2006). Male violence, stress, and neuroendocrine parameters in pregnancy: A pilot study. Biological Research for Nursing, 7, 222-233.

Teixeira, J., Martin, D., Prendiville, O., \& Glover, V. (2005). The effects of acute relaxation on indices of anxiety during pregnancy. Journal of Psychosomatic Obstetrics and Gynaecology, 26, 271-276.

Urech, C., Fink, N. S., Hoesli, I., Wilhelm, F. H., Bitzer, J., \& Alder, J. (2010). Effects of relaxation on psychobiological wellbeing during pregnancy: A randomized controlled trial. Psychoneuroendocrinology, 35, 1348-1355.

Van den Bergh, B. R., Mulder, E. J., Mennes, M., \& Glover, V. (2005). Antenatal maternal anxiety and stress and the neurobehavioural development of the fetus and child: Links and possible mechanisms. A review. Neuroscience and Biobehavioral Reviews, 29, 237-258.

Vieten, C., \& Astin, J. (2008). Effects of a mindfulness-based intervention during pregnancy on prenatal stress and mood: Results of a pilot study. Archives of Women's Mental Health, 11, 67-74.

Vythilingum, B. (2008). Anxiety disorders in pregnancy. Current Psychiatry Reports, 10, 331-335.

Watson, D., Clark, L. A., \& Tellegen, A. (1988). Development and validation of brief measures of positive and negative affect: The PANAS scales. Journal of Personality and Social Psychology, 54, 1063-1070.

Weisberg, R. B., \& Paquette, J. A. (2002). Screening and treatment of anxiety disorders in pregnant and lactating women. Women's Health Issues, 12, 32-36. 